\title{
Tunable Meta-Liquid Crystals
}

\author{
Mingkai Liu,* Kebin Fan, Willie Padilla, David A. Powell, Xin Zhang, \\ and Ilya V. Shadrivov
}

Metamaterials are structures that manipulate waves in previously unimagined ways, ${ }^{[1-3]}$ offering control over the amplitude, phase, polarization, and propagation direction of waves. This is enabled by the carefully controlled properties of the "meta-atom" building blocks. The arrangement of these meta-atoms can correspond to a variety of states of solid media, including crystalline, quasi-crystalline, and amorphous:; ${ }^{[4]}$ recently, the idea has been extended from analog to digital metamaterials with programmable units, ${ }^{[7-9]}$ and the arrangement of meta-atoms can have a pronounced influence on the overall electromagnetic response. Liquid forms of metamaterials were also suggested, including theoretical studies of plasmonic metafluids made of nanoclusters, ${ }^{[10]}$ theoretical studies of dumbbell elements, ${ }^{[11]}$ negative index metafluids, ${ }^{[12]}$ experimental demonstration of an isotropic metafluid with a magnetic response, ${ }^{[13]}$ and demonstration of a colloidal metamaterial made from metallic nanorods. ${ }^{[14]}$ Significant achievements have been made recently in the hybridization of liquid crystals and synthesized colloidal particles, ${ }^{[15-18]}$ making them promising candidates for optical applications. ${ }^{[11,14,19-21]}$ While these techniques based on bottomup approaches are most suitable for optical frequencies, they face challenges in other frequency bands such as the terahertz (THz) regime due to the scalability of the methods. The simple shapes fabricated with bottom-up approaches also set a limitation in the design of material response since the electrostatic and electromagnetic properties are closely related. ${ }^{[11,14]}$

Here, we propose the concept of a tunable liquid metamaterial (Figure $1 \mathrm{~b}$ ). The liquid nature of such metamaterials allows them to flow and fill in spaces or cover surfaces of arbitrary 3D shapes (Figure 1c), which significantly widens the field where metamaterials can be employed. To achieve pronounced tunability, the meta-atoms are grouped into elongated chains and dispersed in a host liquid, and are rotated by electrophoretic forces when an electric field is applied (Figure 1d). This

Dr. M. Liu, Dr. D. A. Powell, Dr. I. V. Shadrivov

Nonlinear Physics Centre

Research School of Physics and Engineering

The Australian National University

Canberra ACT 2601, Australia

E-mail: mingkai.liu@anu.edu.au

Dr. K. Fan, ${ }^{[+]}$Prof. W. Padilla ${ }^{[+]}$

Department of Physics

Boston College

140 Commonwealth Avenue, Chestnut Hill, MA 02467, USA

Dr. K. Fan, Prof. X. Zhang

Department of Mechanical Engineering

Boston University

110 Cummington Mall, Boston, MA 02215, USA

[+]Present address: Department of Electrical and Computer Engineering, Duke University, Box 90291, Durham, NC 27708, USA

DOI: 10.1002/adma.201504924 rotation aligns the axis of anisotropy parallel to the field, tuning the electromagnetic properties of the metamaterial in a manner similar to the mesogens of liquid crystals (Figure 1a). Due to this strong analogy, we call our metamaterial a meta-liquid crystal (MLC) (Figure 1b), and we call the constituent elements meta-mesogens. By changing the direction of the biased electric field, the response of MLCs can be tuned dynamically. In contrast to previous work, the elements of MLCs are complex meta-atoms with subwavelength features and their properties can be engineered in a wide range. Moreover, the electrostatic and electromagnetic properties of our meta-mesogens are weakly correlated, enabling us to construct MLCs with tunable properties not available with conventional liquid crystals, ${ }^{[22]}$ such as large anisotropy, strong magnetic and/or chiral (and in general bianisotropic) responses, or well-designed spectral features such as electromagnetically induced transparency.

While the concept could be realized over a wide range of frequencies, here we experimentally demonstrate the idea in $\mathrm{THz}$ frequency range. Metamaterials play a vital role in controlling THz waves and creating passive and tunable devices, due to the lack of intrinsic response from natural materials. ${ }^{[23-30]}$ A number of solid-state solutions for achieving reconfigurable THz metamaterials have been demonstrated (refs. [31] and [32] and the references therein). These ideas are mostly restricted to 2D platforms; in contrast, the MLCs are intrinsically 3D and freely flowing composites, and thus they represent a new class of tunable metamaterials. They build on previous demonstrations of strong modification of the electromagnetic properties through change of the mutual or internal arrangement of meta-atoms. ${ }^{[33-40]}$

In this work, we design meta-mesogens with a purely electric response, however, it is clear that by adding magnetic meta-atoms one can achieve similar effects with the magnetic response. We chose two types of meta-atoms, electric splitring resonators (ESRRs) and I-beam resonators (IBRs). Each meta-mesogen consists of a short array of identical metallic units encapsulated within $10 \mu \mathrm{m}$ thick polyimide, so that the whole meta-mesogen functions as an anisotropic dipole in an external bias field (see Figure 1b,d). The response of the chosen meta-atoms to the high frequency field is strongly anisotropic, therefore by rotating the whole meta-mesogen, we can tune the overall response of the composite medium.

Here, we study three designs - meta-mesogens made of three ESRRs (denoted as 3-ESRR below), five ESRRs (5-ESRR), and two IBRs (2-IBR) (see Figure 2b and Figure S1 in the Supporting Information for detailed design parameters). Each type of meta-mesogen is mixed in a nonpolar liquid so that they are free to rotate in response to an external bias electric field. Paraffin oil is used in the experimental work as it is a readily available liquid which is safe to handle and transparent in terahertz. 
(a) Liquid Crystals

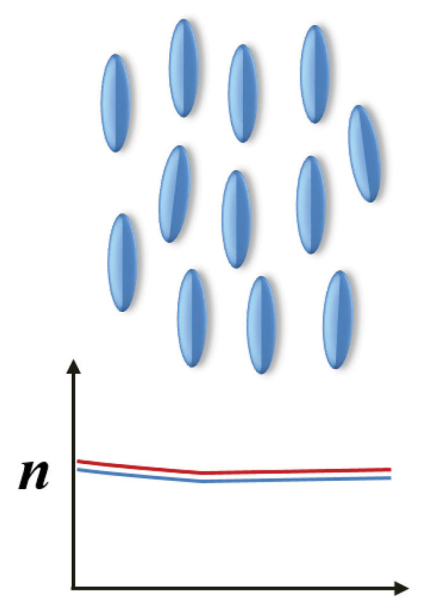

$\omega$ (b) Meta Liquid Crystals

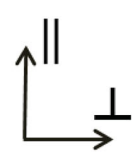

$n \perp$
$n_{\|}$

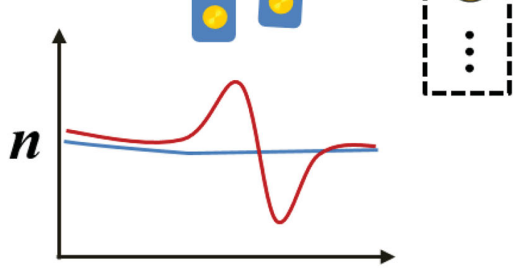

$\omega$
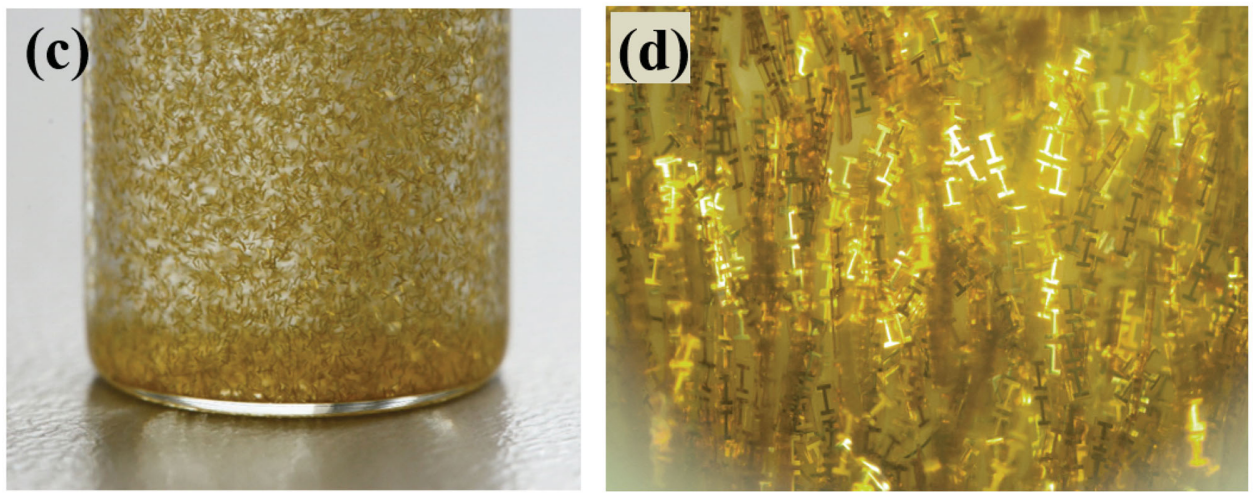

Figure 1. Schematics and corresponding anisotropic refractive index components of a) a conventional liquid crystal in the nematic phase and b) meta-liquid crystals with meta-mesogens oriented along the external static electric field. The electromagnetic properties of the MLC are defined by meta-atoms, while the size and shape of the meta-mesogens largely define the response to the control electric field. The directions parallel and perpendicular to the long axis of mesogens are denoted with "Il" and " $\perp$," respectively. c) Photograph of the fabricated liquid metamaterial in a glass bottle. d) Microscopic image of meta-mesogens aligned under external bias electric field.

The electrostatic torque of a meta-mesogen in a uniform static electric field is well described within the dipole approximation (see Figure S2 and related discussion in the Supporting Information). The torque is primarily determined by the length and aspect ratio of the meta-mesogens. From the torque, we can calculate the response time of meta-mesogens in different liquids. In paraffin oil, all three designs show estimated response times of around $100 \mathrm{~ms}$ for a bias field strength of $1.6 \times 10^{5} \mathrm{~V} \mathrm{~m}^{-1}$ (see Figure S2b in the Supporting Information for details).

While the electrostatic response is similar for the different types of meta-mesogens that we consider, their $\mathrm{THz}$ responses are very different. The THz response of the structures is simulated in a paraffin oil environment, and the geometry of the meta-atoms is tuned so that their lowest order resonances are at $0.5 \mathrm{THz}$. Although they are all electric resonances, their properties are distinct from each other due to the different resonant current profiles for ESRRs and IBRs (see the Supporting Information for more details). The MLCs based on ESRRs are expected to have sharper resonances due to the circulating current of the mode, while IBRs have a broader resonance due to strong dipole radiation and can provide a more pronounced anisotropy. According to the calculations, one unique feature of the designed MLCs is that they can exert strong modulation on the transmitted signal even though the volume density of metamesogens in the composite is very low (less than 1\%). The volume density here is defined as the volume of all meta-mesogens (polyimide + metal) over the volume of the meta-liquid composites (meta-mesogens + host liquid). This strong amplitude modulation is a distinct feature compared with conventional liquid crystals that mainly modulate the phase. For more details on the theoretical and numerical calculation of the $\mathrm{THz}$ response of the designed meta-mesogens, see the Supporting Information.

To verify that the MLCs exhibit the predicted dynamics and morphology transitions, we fabricated the designed meta-mesogens with a standard UV lithography process on silicon handling wafers (Figure 2a (1) to (5)). After fabrication, the metamesogens are lifted off by dissolving the sacrificial layers in acetone and then air-dried (Figure 2a (6)). The meta-mesogens 
(a)

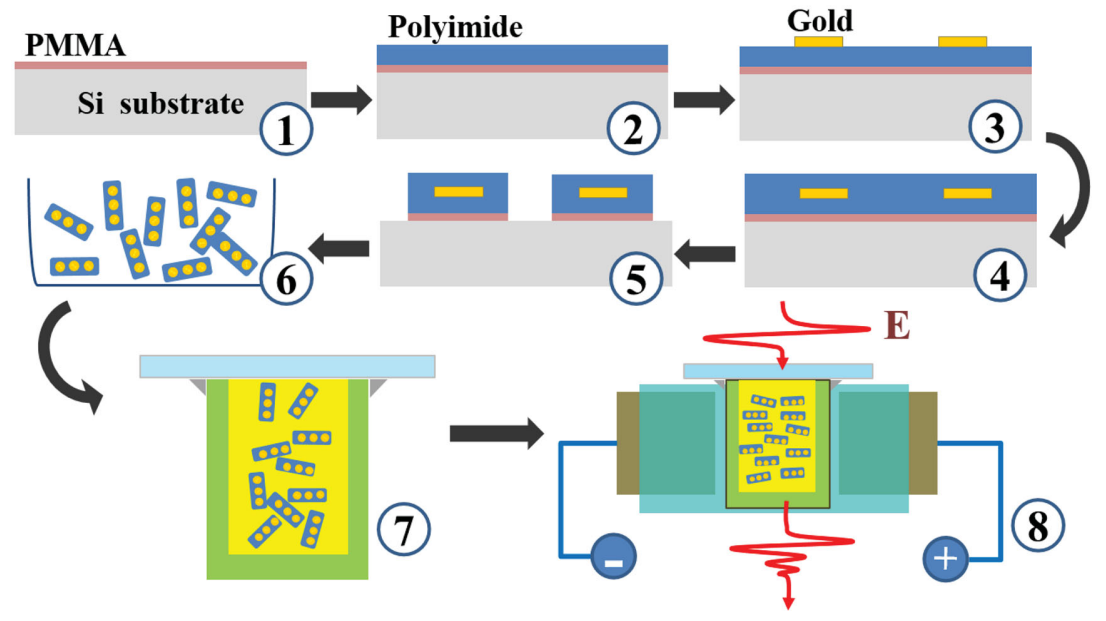

(b)

3-ESRR

5-ESRR

2-IBR
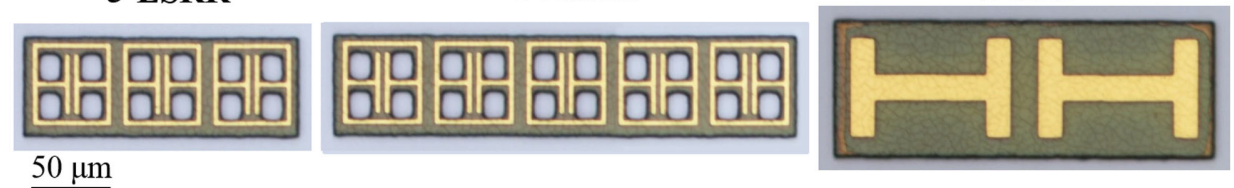

Figure 2. Sample preparation. a) Procedure of meta-mesogen fabrication and preparation of meta-liquid crystal sample. (1) PMMA sacrificial layer coating, (2) polyimide coating and curing, (3) meta-atom patterning, (4) polyimide encapsulating, (5) polyimide etching, (6) meta-mesogen releasing and drying, (7) mixture of meta-mesogens and paraffin oil injected into sample chamber and sealed with quartz slides, and (8) spectral measurement under bias electric field. b) Microscope photographs of the fabricated meta-mesogens.

are then mixed with paraffin oil and injected into the polytetrafluoroethylene (PTFE) cylindrical cell ( $5 \mathrm{~mm}$ outer diameter, $3 \mathrm{~mm}$ inner diameter, $3.5 \mathrm{~mm}$ depth) and sealed with a quartz plate (Figure 2a (7)) so that we can observe the behavior of the meta-liquid crystals under an optical microscope. The whole sample cell is fixed into a PTFE holder with integrated pair of electrodes connected to the external bias voltage (see Figure 2a (8)). When a voltage is applied to the electrodes, the metamesogens experience strong torque and reorient along the electric field. The original randomly oriented meta-mesogens transform into well-aligned states (see Figure 3). Note that the current electrode arrangement only allows alignment of the meta-mesogens along one axis; the control electrodes would need to be redesigned and optimized for the meta-mesogens to be dynamically switchable between orthogonal directions.

The dynamic response and morphology are affected by the concentration of meta-mesogens. When the concentration is low, they interact less with each other and are free to move around in the paraffin oil. Once the bias voltage is applied, they redirect along the electric field and gradually aggregate
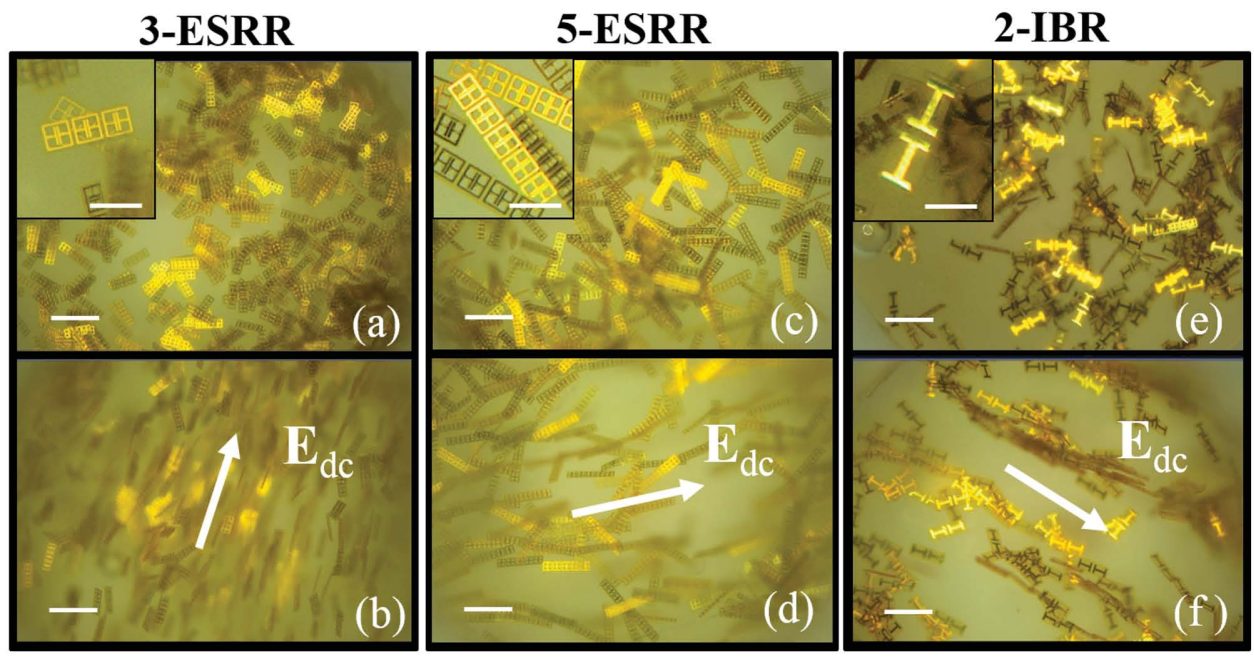

Figure 3. Morphology change of the three different meta-mesogens before (top row) and $5 \mathrm{~s}$ after (bottom row) application of the bias electric field a,b) 3-ESRR, c,d) 5-ESRR, and e,f) 2-IBR. The scale bars in the main figures and insets are 300 and $100 \mathrm{~mm}$, respectively. 
into elongated clusters; some of the meta-mesogens connect to each other to form long chains (see Figure 3d,f, which shows a lower number density of around $15 \mu \mathrm{L}^{-1}$ ), while the others may stick to the walls of the cell close to the two electrodes. For higher concentration, the meta-mesogens interact more with each other and the effective viscosity increases. In such cases, as long as the voltage is not applied for a long time, the meta-mesogens are more prone to change their orientation with minimal change in their position (see Figure 3b, having a higher number density of around $32 \mu \mathrm{L}^{-1}$ ).

We note that since the paraffin is less dense than the mesogens, they slowly sink, and the relatively high viscosity limits switching speeds. Further search for more suitable host liquids is required, and as an example, in the Supporting Information we present the results of simulations for trichloroethylene. It is less viscous and denser than paraffin oil, making it more suitable for a faster performing MLC. Our calculations predict that this can provide at least one order of magnitude enhancement of the operation speed. Trichlorethylene was not used in our experiments due to its toxicity. The dynamics can further be optimized by choosing the shape and size of the meta-mesogens. Since the sign of the voltage output from our source cannot be changed, there is a net DC contribution leading to charge build up, which would result in the aggregation of meta-mesogens, therefore bursts of pulses are used to maintain the alignment (see the Supporting Information). To enable practical application of this idea, further technical optimization is required so that the charging effect and sinking problem can be addressed.

To quantify the anisotropy induced in the $\mathrm{THz}$ spectral response, the transmission is measured with a commercial $\mathrm{THz}$ time-domain spectroscope (EKSPLA). In the measurement, we rotate the sample with respect to the polarization of the incident wave to characterize the phase and amplitude modulation in the parallel and perpendicular directions. Figure $4 a-c$ shows the transmittance spectra $|t|^{2}$ for three different samples. Note that the transmission curves are normalized to the free space transmission in order to demonstrate the performance of the whole device. We performed multiple measurements and the maximum relative errors are around $20 \%$, and they are omitted in Figure 4 for clarity (see Figure S6 in the Supporting Information for details). The transmission is affected both by losses in the structure and by reflection. The most profound effect is due to reflection at the air/quartz interface from the input side, the PTFE/air interface from the output side, as well as the scattering and absorption from the wall of PTFE cell (the $\mathrm{THz}$ beam width is around $4 \mathrm{~mm}$ at $0.5 \mathrm{THz}$, larger than the inner diameter of the cell), with only around $1 / 3$ of the energy
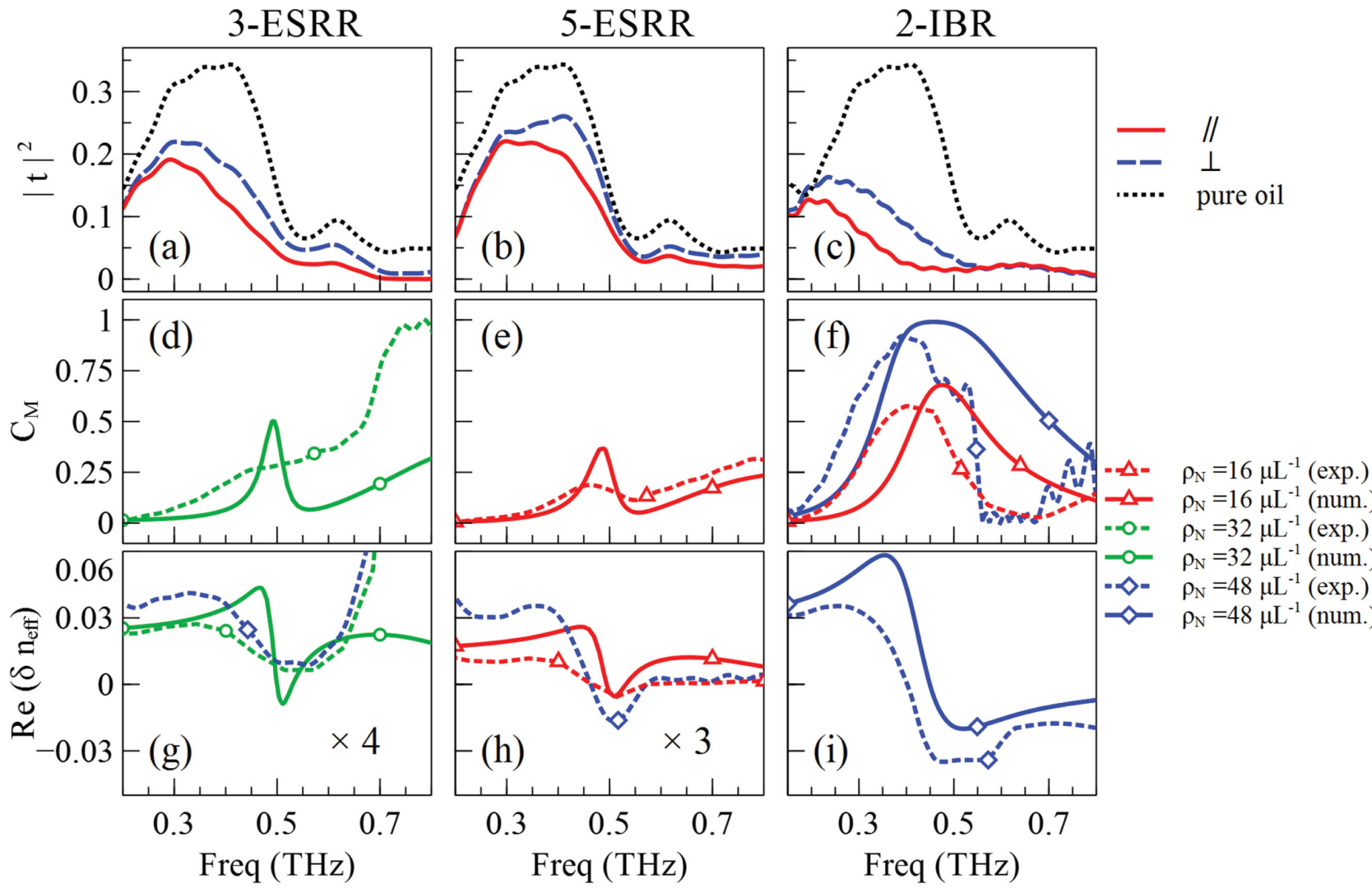

Figure 4. a-c) Measured transmittance spectra of three different types of meta-mesogens for orthogonal polarizations. The corresponding particle numbers for 3-ESRR, 5-ESRR, and 2-IBR are around 800, 370, and 1140, respectively. Spectra of pure paraffin oil are also given as a reference. $d-f$ ) Modulation contrast of transmission under different number density. The spectra plotted with short-dashed lines and solid lines are measured and numerical results, respectively. g-i) Effective index difference calculated from the relative transmission phase difference for orthogonal polarizations. The blue dotted curves in $(\mathrm{g})$ and $(\mathrm{h})$ are normalized results to the same number of meta-mesogens $N=1200$, corresponding to a number density of $\rho_{N}=48 \mu \mathrm{L}^{-1}$. 
left when the signal transmits through an empty cell. Another contribution is due to absorption of energy by the paraffin oil. The oil is quite transparent below $0.5 \mathrm{THz}$, and the loss increases as frequency goes up. We believe the transmission of the device can be improved by reducing reflection at the input interface with a proper antireflection coating, and by finding a more appropriate liquid with lower absorption. The modulation contrasts, defined as $C_{M}=\left(\left|t_{\perp}\right|^{2}-\left|t_{\|}\right|^{2}\right) /\left(\left|t_{\|}\right|^{2}+\left|t_{\perp}\right|^{2}\right)$, show that the modulation effect is quite noticeable (see Figure $4 d-f$ ). It is interesting to note that the trends in modulation contrast are different for the designs with ESRRs and IBRs. For the two samples with ESRRs, there is an increasing trend as frequency increases, and this is consistent with the growing trend predicted in the calculated extinction cross section (see Figure S3g,h in the Supporting Information). As a comparison, we plot the theoretical results calculated based on the scattering properties of the meta-mesogens. In the calculation, we assume that the meta-mesogens form a cubic lattice and use ClausiusMossotti formula to estimate the effective permittivity under different number density; the results are further employed to calculate the transmission spectra (see the Supporting Information for more details). The modeled results show overall good agreement apart from some redshifts of the measured resonances. This is primarily because the theoretical model is based on the point dipole approximation, and we assume that the dipoles are homogenously distributed; but in practice the meta-mesogens have finite length and they can form complicated hierarchal structures such as long chains and clusters due to the complicated electrostatic and hydrodynamic interaction. This effectively brings in stronger electromagnetic interaction among meta-mesogens and leads to a redshift as compared to the ideal situation simulated in the model (see the Supporting Information for more discussion).

For sample 3-ESRR, we used a larger concentration $\left(N \approx 800, \rho_{N} \approx 32 \mu \mathrm{L}^{-1}\right)$ due to its smaller number of effective meta-atoms. Yet, due to the inherently smaller anisotropy and the partially nonuniform orientation of meta-mesogens, the resonant peak around $0.5 \mathrm{THz}$ is less noticeable (see Figure $4 \mathrm{~d}$ ). Interestingly, the strong peak around $0.78 \mathrm{THz}$ is not expected from simulations since the collective dipole resonances of a single meta-mesogen should occur above $1 \mathrm{THz}$. This resonant feature could be due to multiple scattering or intermesogen interaction and requires further investigation. To compare the modulation contrast of IBR and ESRR, we first used similar numbers of meta-mesogens for sample 5-ESRR $(N \approx 370)$ and sample 2-IBR $(N \approx 400)$, and we found that the modulation contrast of sample 2-IBR is approximately twice the value of 5-ESRR (see red short-dashed curves marked with triangles in Figure $4 \mathrm{e}, \mathrm{f}$ ) which is in agreement with the predictions from simulations (see Figures S3 and S4 in the Supporting Information). After confirming that 2-IBR has the strongest modulation of the three designs, we increased its number to $N \approx 1140$ $\left(\rho_{N} \approx 48 \mu \mathrm{L}^{-1}\right)$ and a significant modulation contrast larger than 0.9 was observed around the resonance (see blue short-dashed line marked with diamonds in Figure 4f). The observed effects are reproduced qualitatively well with theory.

From the measured transmission phase, we calculate the effective linear birefringence $\delta n_{\text {eff }} \approx \arg \left(t_{\|} / t_{\perp}\right) /\left(k_{0} h\right)$ produced by the three different designs, where $k_{0}=\omega / c$ and $h$ is the thickness of the MLC sample (see Figure $4 \mathrm{~g}-\mathrm{i}$ ). To have a fair comparison, we normalize the index to the same mesogen number of $N=1200, \rho_{N}=48 \mu L^{-1}$ (see blue short-dashed lines with diamonds from Figure $4 \mathrm{~g}-\mathrm{i}$ ). This normalization is based on the linear approximation of the relation between effective index and number density of mesogens $n_{\text {eff }} \propto N$, and should be valid when the concentration is low. The resonant features for all three samples are clearly observed, and the effective index difference of sample 2-IBR is about three times the value of 5-ESRR. Compared with the calculation for the same number density (solid lines in Figure 4g-i), the index differences are about $1 / 3$ to $1 / 2$ of the predicted values. Several factors can contribute to the reduced value, such as nonuniformity in the orientation and distribution of the meta-mesogens in the experiment. Nevertheless, our results provide qualitatively good agreement with the predicted effects, showing that the electromagnetic response of MLCs can be well prescribed.

We emphasize that the volume concentrations of meta-mesogens used in the experiment are very low (less than 1\%), thus the real index difference (proportional to transmission phase difference) around the resonance is smaller than conventional liquid crystals. ${ }^{[22]}$ On the other hand, the modulation contrast of transmission amplitude is significant due to the strong scattering of meta-atoms that leads to reflection, in contrast to natural liquid crystals which usually only modulate phase. Stronger anisotropy can be achieved by increasing the concentration, but it could lead to stronger interaction between meta-mesogens and a balance between response time and operating voltage is required. Alternatively, it would be more favorable to use meta-mesogens with ultrastrong anisotropic scattering properties that simultaneously support electric and magnetic multipole resonances.

In summary, we present an experimental study of tunable 3D THz meta-liquid crystals, where engineered meta-mesogens play a similar role to the mesogens of a natural liquid crystal. The most important feature of meta-liquid crystals is that the electromagnetic and electrostatic response can be independently engineered, which provides a much greater flexibility in constructing tunable metamaterials and meta-devices. We fabricated samples based on different designs of electric metaatoms, and they showed the designed anisotropy with distinctive spectral features. Different from conventional liquid crystals, the meta-liquid crystals studied here can provide a direct and pronounced amplitude modulation of transmission. We believe that MLCs can also be made sensitive to a bias magnetic field by the use of suitable substrates in the meta-mesogens. One could also consider using prepolarized materials in the meta-mesogens in order to reduce the bias electric field required to tune the structure. The meta-atoms can be fabricated in a wide variety of shapes, and could even be made from high index dielectric resonators, which are appealing due to their low losses at optical wavelengths. We expect that with advances in nanofabrication, meta-liquid crystals could be fabricated on a length scale suitable for applications in optics.

\section{Supporting Information}

Supporting Information is available from the Wiley Online Library or from the author. 


\section{Acknowledgements}

M.L., D.A.P., and I.V.S. were supported by the Australian Research Council. K.F. and X.Z. were supported by the National Science Foundation (ECCS-1309835). K.F. and W.P. were supported by the Office of Naval Research (N00014-15-1-0051).

Received: October 6, 2015

Revised: November 9, 2015

Published online: December 8, 2015

[1] J. B. Pendry, D. Schurig, D. R. Smith, Science 2006, 312, 1780

[2] D. R. Smith, N. Kroll, Phys. Rev. Lett. 2000, 85, 2933.

[3] E. E. Narimanov, A. V. Kildishev, Appl. Phys. Lett. 2009, 95, 041106.

[4] C. Helgert, C. Rockstuhl, C. Etrich, C. Menzel, E. B. Kley, A. Tünnermann, F. Lederer, T. Pertsch, Phys. Rev. B 2009, 79, 233107.

[5] M Albooyeh, D. Morits, S. Tretyakov, Phys. Rev. B 2012, 85, 205110.

[6] S. S. Kruk, C. Helgert, M. Decker, I. Staude, C. Menzel, C. Etrich, C. Rockstuhl, C. Jagadish, T. Pertsch, D. N. Neshev, Y. S. Kivshar, Phys. Rev. B 2013, 88, 201404.

[7] C. Della Giovampaola, N. Engheta, Nat. Mater. 2014, 13, 1115.

[8] T. J. Cui, M. Q. Qi, X. Wan, J. Zhao, Q. Cheng, Light: Sci. Appl. 2014, 3, e218.

[9] L. H. Gao, Q. Cheng, J. Yang, S. J. Ma, J. Zhao, S. Liu, H. B. Chen, Q. He, W. X. Jiang, H. F. Ma, Q. Y. Wen, L. J. Liang, B. B. Jin, W. W. Liu, L. Zhou, J. Q. Yao, P. H. Wu, T. J. Cui, Light: Sci. Appl. 2015, 4, e324.

[10] Y. A. Urzhumov, G. Shvets, J. A. Fan, F. Capasso, D. Brandl, P. Nordlander, Opt. Express 2007, 15, 14129.

[11] A. A. Zharov, A. A. Zharov, N. A. Zharova, J. Opt. Soc. Am. B 2014, $31,559$.

[12] M. Fruhnert, S. Mühlig, F. Lederer, C. Rockstuhl, Phys. Rev. B 2014, 89, 075408.

[13] S. N. Sheikholeslami, H. Alaeian, A. L. Koh, J. A. Dionne, Nano Lett. 2013, 13, 4137.

[14] A. B. Golovin, O. D. Lavrentovich, Appl. Phys. Lett. 2009, 95, 254104.

[15] M. R. Jones, R. J. Macfarlane, B. Lee, J. Zhang, K. L. Young, A. J. Senesi, C. A. Mirkin, Nat. Mater. 2010, 9, 913.

[16] M. R. Jones, K. D. Osberg, R. J. Macfarlane, M. R. Langille, C. A. Mirkin, Chem. Rev. 2011, 111, 3736.

[17] H. K. Bisoyi, S. Kumar, Chem. Soc. Rev. 2011, 40, 306.
[18] J. P. Lagerwall, G. Scalia, Curr. Appl. Phys. 2012, 12, 1387.

[19] Q. Liu, Y. Cui, D. Gardner, X. Li, S. He, I. I. Smalyukh, Nano Lett. 2010, 10, 1347.

[20] D. Coursault, J. Grand, B. Zappone, H. Ayeb, G. Lévi, N. Félidj, E. Lacaze, Adv. Mater. 2012, 24, 1461.

[21] J. Dintinger, B. J. Tang, X. Zeng, F. Liu, T. Kienzler, G. H. Mehl, G. Ungar, C. Rockstuhl, T. Scharf, Adv. Mater. 2013, 25, 1999.

[22] H. Park, E. P. J. Parrott, F. Fan, M. Lim, H. Han, V. G. Chigrinov, E. Pickwell-MacPherson, Opt. Express 2012, 20, 11899.

[23] T. J. Yen, W. Padilla, N. Fang, D. Vier, D. Smith, J. Pendry, D. Basov, X. Zhang, Science 2004, 303, 1494.

[24] W. Padilla, M. Aronsson, C. Highstrete, M. Lee, A. Taylor, R. Averitt, Phys. Rev. B 2007, 75, 041102.

[25] H. Tao, N. I. Landy, C. M. Bingham, X. Zhang, R. D. Averitt, W. J. Padilla, Opt. Express 2008, 16, 7181.

[26] O. Paul, C. Imhof, B. Reinhard, R. Zengerle, R. Beigang, Opt. Express 2008, 16, 6736.

[27] G. P. Williams, Rep. Prog. Phys. 2006, 69, 301.

[28] H. T. Chen, J. F. O'Hara, A. K. Azad, A. J. Taylor, Laser Photonics Rev. 2011, 5, 513

[29] M. Tonouchi, Nat. Photonics 2007, 1, 97.

[30] C. Watts, D. Shrekenhamer, J. Montoya, G. Lipworth, J. Hunt, T. Sleasman, S. Krishna, D. Smith, W. Padilla, Nat. Photonics 2014, 8,605 .

[31] N. I. Zheludev, Y. S. Kivshar, Nat. Mater. 2012, 11, 917.

[32] K. Fan, W. J. Padilla, Mater. Today 2015, 18, 39

[33] H. Tao, A. Strikwerda, K. Fan, W. Padilla, X. Zhang, R. Averitt, Phys. Rev. Lett. 2009, 103, 147401.

[34] Y. H. Fu, A. Q. Liu, W. M. Zhu, X. M. Zhang, D. P. Tsai, J. B. Zhang, T. Mei, J. F. Tao, H. C. Guo, X. H. Zhang, J. H. Teng, N. I. Zheludev, G. Q. Lo, D. L. Kwong, Adv. Funct. Mater. 2011, 21, 3589.

[35] W. Zhu, A. Liu, T. Bourouina, D. Tsai, J. Teng, X. Zhang, G. Lo, D. Kwong, N. Zheludev, Nat. Commun. 2012, 3, 1274.

[36] J. Y. Ou, E. Plum, J. Zhang, N. I. Zheludev, Nat. Nanotechnol. 2013, 8, 252.

[37] M. Lapine, I. V. Shadrivov, D. A. Powell, Y. S. Kivshar, Nat. Mater. 2012, 11, 30.

[38] A. P. Slobozhanyuk, M. Lapine, D. A. Powell, I. V. Shadrivov, Y. S. Kivshar, R. C. McPhedran, P. A. Belov, Adv. Mater. 2013, 25, 3409.

[39] M. Liu, Y. Sun, D. A. Powell, I. V. Shadrivov, M. Lapine, R. C. McPhedran, Y. S. Kivshar, Phys. Rev. B 2013, 87, 235126.

[40] M. Liu, D. A. Powell, I. V. Shadrivov, M. Lapine, Y. S. Kivshar, Nat. Commun. 2014, 5, 4441. 